\section{Contamination of dropper bottles with tear fluid in an ophthalmic outpatient clinic}

The isolation of the human immunodeficiency virus (HIV) from the tears of a patient with the acquired immune deficiency syndrome (AIDS) ${ }^{1}$ has aroused concern about the risks of transmission of the virus during various ophthalmic procedures. ${ }^{2}$ Current recommendations permit the use of multiple application dropper bottles in ophthalmic outpatient departments, though opened bottles should be discarded at the end of each day. ${ }^{3} \mathrm{We}$ performed a simple study to discover whether such bottles can become contaminated with tear fluid during normal use.

\section{Methods and results}

The study took place during a single ophthalmic outpatient clinic. Oxybuprocaine (Benoxinate) was supplied in glass multiple application dropper bottles incorporating a dropper in the screw cap. One bottle was provided for each of eight slit lamps. For tonometry we asked that a drop of oxybuprocaine should be instilled into the tear sac after $1 \%$ fluorescein, thus reversing the usual order of application. To eliminate bias and otherwise maintain normal practice we did not explain the purpose of the study to the medical staff until afterwards. At the end of the clinic the eight bottles were collected and examined for contamination with fluorescein by using a Perkin Elmer 3000 fluorescence spectrometer set for an excitation frequency of $440 \mathrm{~nm}$ and an emission frequency of $510 \mathrm{~nm}$. The instrument was calibrated by using serial dilutions of $1 \%$ fluorescein. The volume of drops remaining in each bottle was also measured as a rough guide to the number of times each bottle had been used.

The table shows that six of the eight bottles were contaminated with fluorescein. The bottles that were used more frequently tended to have heavier contamination.

Contamination of dropper bottles with fluorescein

\begin{tabular}{lcc}
\hline Bottle No & $\begin{array}{c}\text { Volume }(\mathrm{ml}) \\
\text { (to nearest } 0 \cdot 25 \mathrm{ml})\end{array}$ & $\begin{array}{c}\text { Contamination } \\
\text { (nl equivalent of } \\
\text { 1\% fluorescein) }\end{array}$ \\
\hline Control & $4 \cdot 0$ & 0 \\
1 & $3 \cdot 75$ & 0 \\
2 & $3 \cdot 5$ & 1 \\
$3^{\star}$ & $2 \cdot 75$ & 10 \\
4 & $3 \cdot 0$ & 5 \\
5 & $3 \cdot 25$ & 6 \\
$6^{\star}$ & $2 \cdot 5$ & 38 \\
7 & $3 \cdot 0$ & 4 \\
8 & $3 \cdot 75$ & 0 \\
\hline
\end{tabular}

^ Fluorescein contamination of these bottles was detectable with the naked eye.

\section{Comment}

Our study shows that dropper bottles can be contaminated with the content of tear fluid, in contradistinction to other sources, within a single ophthalmic outpatient clinic. There are various ways in which this could occur. If the drop is touched rather than dropped on to the conjunctiva there may be reflux into the dropper. This is particularly likely if the drop is applied while the patient's chin is resting on the slit lamp. If the dropper is held too low and the patient's head is not sufficiently far back the tip of the dropper may brush against the lashes of the upper lid.

The practical importance of this result relates to patients without obvious external eye disease who none the less have microbes in their tears. Commensal bacteria are present in the conjunctival sac, and bacterial contamination of dropper bottles may occur. ${ }^{4}$ For this reason preservatives are used. Contaminated ophthalmic solutions have also been responsible for the spread of viral disease, as in an outbreak of infection with adenovirus type 8 in Alabama. ${ }^{5}$

HIV has been isolated from the tears of a patient with AIDS, ${ }^{\prime}$ but the extent to. which it is present and active in the tears of the much larger group of asymptomatic carriers is not known. We know of no evidence to suggest that HIV has been transmitted through contact with tears. If a dropper bottle did become contaminated and if virus was then instilled into the conjunctival sac of a patient it is still unlikely that infection would occur. An analogy would be contact with saliva, which also contains HIV. Studies of exposed hospital staff, family members, and sexual partners of infected individuals have failed to show transmission of the virus by any medium other than blood and semen.

Thus contamination of multiple application dropper bottles with tear fluid may occur even when the current recommendations are complied with. Greater care or the use of single application packs would eliminate the small but finite risk that such contamination represents.

We thank Mr Redmond J H Smith for valuable advice and for allowing us to conduct the study during his outpatient clinic, and Mr A E Rippon, department of chemical pathology, St Mary's Hospital Medical School, London, for help with the fluorescence spectrometer.

1 Fujikawa LS, Salahuddin SZ, Palestine AG, Masur H, Nussenblatt RB, Gallo RC. Isolation of human T-lymphotropic virus type III from the tears of a patient with the acquired immunodeficiency syndrome. Lancet 1985;ii:529-30.

2 Anonymous. Leads from the MMWR. JAMA 1985;254:1429.

3 Anonymous. Eye-drops. The pharmaceutical codex. 11th ed. London: Pharmaceutical Press, 1979:346-9.

4 Aslund B, Olson OT, Sandell E. Studies on in-use contamination of eye drops. Acta Pharm Suec 1978;15:389-94.

5 Sprague JB, Hierholzer JC, Currier RW, Hattwick MAW, Smith MD. Epidemic keratoconjunctivitis. A severe industrial outbreak due to adenovirus type 8. N Engl f Med 1973;289:1341-6.

(Accepted 5 March 1987)

Western Ophthalmic Hospital, London NW1 5 YE

G W AYLWARD, MB, BCHIR, senior house officer

R S WILSON, FRCS, senior registrar

Correspondence to: $\mathrm{Mr}$ Wilson.

\section{Limitations of direct ophthalmoscopy in screening for glaucoma}

Primary open angle glaucoma is one of the most common causes of visual loss encountered in clinical practice. Some form of screening is needed so that ophthalmologists can see and treat patients before they develop symptoms and reach an advanced stage of the disease. At present, most screening is performed by opticians, who examine fundi and occasionally assess intraocular pressures and visual fields of patients. Theoretically, general practitioners are better placed to identify patients at risk of developing glaucoma; however, they rely solely on direct ophthalmoscopy in screening for glaucoma. ${ }^{1}$ Stereophotographs of optic discs may be used to predict glaucomatous defects in the visual field, with a specificity and sensitivity of over $90 \% .{ }^{2}$ To find out whether direct ophthalmoscopy can match this we conducted a survey at a recent North of England Ophthalmological meeting in which we checked the ability of ophthalmologists to screen for glaucoma by direct ophthalmoscopy alone.

\section{Method}

Thirty four ophthalmologists participated in the study, 20 of whom were consultants. Twenty two patients aged between 32 and 75 were examined. One patient had a unilateral vitreous haemorrhage so there were only 43 discs to assess. Sixteen discs were in eyes which had definite early or moderate glaucomatous defects in the visual field. The other 27 discs were in eyes which did not have any demonstrable glaucomatous defect on Goldman perimetry. This group consisted of eyes of normal subjects or patients with ocular hypertension and the normal eyes of patients with unilateral glaucoma.

Patients' eyes were dilated with tropicamide $0.5 \%$ before examination in a darkened room. Doctors' assessments were independent of one another, each doctor filling in an answer sheet by ticking "yes", "no", or "unsure" for each disc. The scores for unsure were not included in either the false positive or the false negative percentages or the specificity or sensitivity percentages.

The junior doctors diagnosed more discs as glaucomatous (table), indicating not only a better detection rate for glaucoma but also a higher false positive score. In both groups the sensitivity and, to a less degree, the specificity were less than that reported with stereophotographs. In addition, there was considerable interobserver disagreement on the glaucomatous discs.

\section{Comment}

Direct ophthalmoscopy of the optic disc entails assessing a monocular view of a sometimes highly mobile target. This study has shown that, when 